\title{
SPACE CURVES THAT POINT ALMOST EVERYWHERE
}

\author{
BY
}

J. B. WILKER

\begin{abstract}
We construct a simple, closed, continuously differentiable curve $r:[0,1] \rightarrow E^{d}(d>3)$ whose tangent vector never points twice in the same direction of $S^{d-1}$ yet sweeps out a set of directions equal to almost all of $S^{d-1}$.
\end{abstract}

1. Introduction. The circle is a simple, closed, continuously differentiable curve in $E^{2}$ whose tangent vector points exactly once in every direction of $S^{1}$. No curve in $E^{d}(d \geqslant 3)$ can possess all of the analogous properties but there are examples which come surprisingly close to doing so.

THEOREM 1. There exists a simple, closed, continuously differentiable curve in $E^{d}(d \geqslant 3)$ whose tangent vector points at least once in every direction of $S^{d-1}$. The tangent vector to any such curve must point at least $d$ times in certain directions. In fact this is true independent of the curve being simple or closed.

THEOREM 2. Consider the continuously differentiable curves in $E^{d}(d \geqslant 3)$ whose tangent vector never points twice in the same direction of $S^{d-1}$ and therefore omits certain directions. If the tangent vector to such a curve is forbidden to vanish, then its set of omitted directions must have positive $(d-1)$-dimensional measure. However, there exist simple closed curves of this type whose set of omitted directions has arbitrarily small $(d-1)$-dimensional measure. On the other hand, if the tangent vector is allowed to vanish countably often, there is a simple, closed curve whose set of omitted directions has $(d-1)$-dimensional measure zero.

It is natural to ask if there exists a simple, closed, differentiable curve in $E^{d}$ $(d \geqslant 3)$ whose discontinuously varying tangent vector points exactly once in every direction of $S^{d-1}$. This question remains open.

The construction for Theorem 1 is achieved by splicing integrals of Peano curves, and the remark about multiplicity is proved by applying a result of Lebesgue. The constructions for Theorem 2 are achieved by splicing integrals of Osgood curves, but here the splicing is more delicate and in the second case a preliminary application of the Vitali covering theorem is required.

Received by the editors December 30, 1977 and, in revised form, April 14, 1978.

AMS (MOS) subject classifications (1970). Primary 54F25; Secondary 53A05, 28A75.

Key words and phrases. Peano curve, Osgood curve, Vitali covering theorem. 
Peano and Osgood curves are developed in $\S 2$. Theorem 1 is proved in $\S 3$ and Theorem 2 is proved in $\S \S 4$ and 5. Related work is discussed in $\S 6$.

2. Peano and Osgood curves. In 1890, Peano [7] startled the mathematical community by constructing his continuous curves $p:[0,1] \rightarrow[0,1]^{n}(n \geqslant 2$ and especially $n=2,3$ ) which cover $[0,1]^{n}$. The description of these curves was arithmetic in the sense that the first $\mathrm{nm}$ ternary "decimals" of $t$ were manipulated to give the first $m$ ternary decimals of each of the $n$ components of $p(t)$. In effect $t$ was identified with a nest of closed sets in [0, 1], hence with a nest of closed sets in $[0,1]^{n}$ and hence with $p(t)$. Figure 1 indicates a geometric interpretation of Peano's original construction for $n=2$ by showing how to match successively refined partitions of $[0,1]$ and $[0,1]^{2}$ into $3^{m}$ closed sets $(m=1,2,3,4)$. In 1891, Hilbert [3] underlined the geometric meaning of Peano's construction and drew pictures analogous to Figure 1 for partitions of $[0,1]$ and $[0,1]^{2}$ into $4^{m}$ closed sets $(m=1,2,3)$.
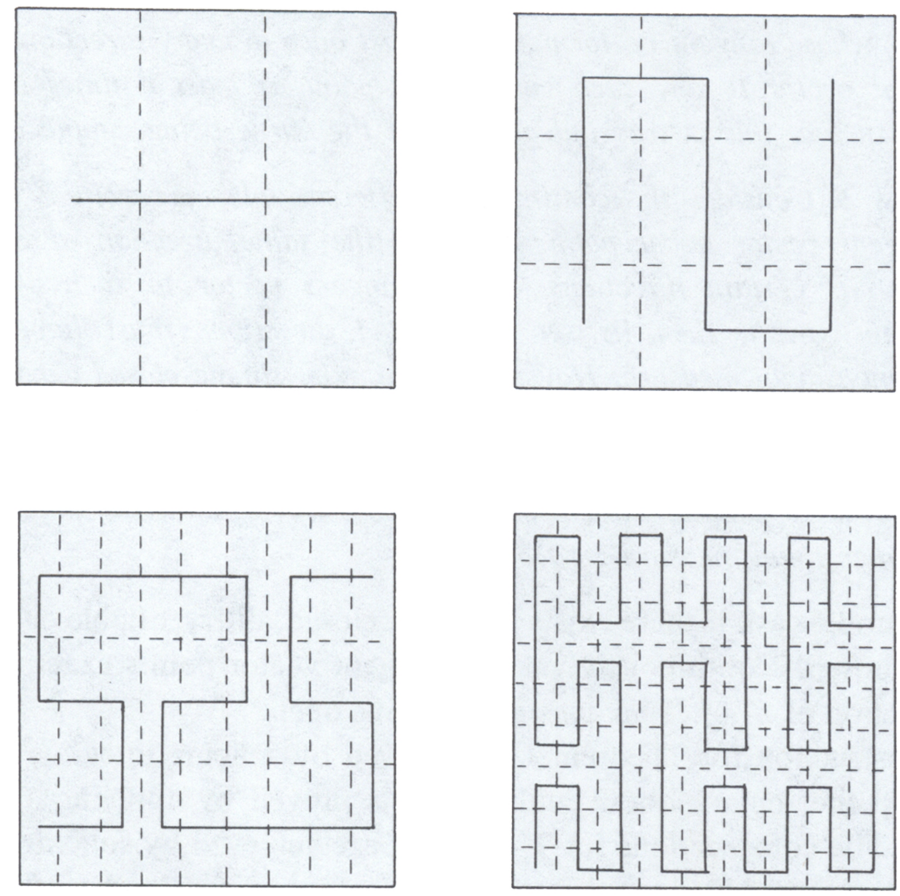

FIGURE 1

A somewhat different arithmetic description of a continuous surjection $p$ : $[0,1] \rightarrow[0,1]^{2}$ runs as follows. If $t$ has the decimal expansion $t$ $=t_{1} t_{2} t_{3} t_{4} \cdots$, let the components of $p(t)$ have decimal expansions $x(t)$ $=. t_{1} t_{3} \cdots$ and $y(t)=. t_{2} t_{4} \cdots$. Although this does not quite work, its only 
essential failing is typified by the fact that $t_{n}^{\prime}=.4999 \cdots(n$ 's $) \rightarrow t=.5$ while $p\left(t_{n}^{\prime}\right)=(.49 \cdots, .99 \cdots) \rightarrow(.5,1) \neq(.5,0)=p(t)$. To correct this type of difficulty, restrict the above definition of $p(t)$ to the closed subset $F$ of $[0,1]$ consisting of those $t$ which have a 9-free decimal expansion. Interpret $x(t)$ and $y(t)$ as numbers in the scale of 9 so that $p(F)$ already covers $[0,1]^{2}$. Finally, extend $p$ to all $[0,1]$ by linear interpolation on the open intervals of $[0,1]-F$. This construction generalizes immediately to give $p:[0,1] \rightarrow$ $[0,1]^{n}$. An extra surprise is that $p(F)=[0,1]^{n}$ even though $F$ has one-dimensional measure zero!

None of the above Peano mappings of $[0,1]$ onto $[0,1]^{n}$ can be $1-1$ because that would make $p^{-1}$ continuous and $[0,1]$ and $[0,1]^{n}$ homeomorphic. This is impossible by the elementary observation that a single point can be removed to disconnect $[0,1]$ but not $[0,1]^{n}$. In fact Lebesgue [5] has shown that for any Peano mapping $p:[0,1] \rightarrow[0,1]^{n}$ there are points $x \in[0,1]^{n}$ for which $p^{-1}(x)$ contains at least $n+1$ points.

In 1903, Osgood [6] showed that a Jordan curve $q:[0,1] \rightarrow[0,1]^{2}$ can have positive area despite the fact that it is homeomorphic to $[0,1]$ and therefore topologically a 1-dimensional set. More generally, it is true that a 1-1 continuous function $q:[0,1] \rightarrow[0,1]^{n}(n \geqslant 2)$ can have the property that $q^{*}=q([0,1]) \subset[0,1]^{n}$ has positive $n$-dimensional Lebesgue measure, $\mu^{n}\left(q^{*}\right)$ $>0$. We offer a proof of this in a form convenient for later use.

LEMMA 1. Let $B$ be a closed ball of radius $r>0$ in $E^{n}(n \geqslant 2)$ and let $\alpha$ be a number satisfying $0<\alpha<1$. Then there is a 1-1 continuous function $q$ : $[0,1] \rightarrow B$ such that $\mu^{n}\left(q^{*}\right)>\alpha \mu^{n}(B)$. It is possible to arrange that $q(0)$ and $q(1)$ lie on the boundary of $B$ and the rest of $q^{*}$ lies in the interior of $B$.

Proof. The proof falls into 4 steps.

STEP 1. Let $B$ be a closed ball of radius $r>0$ in $E^{n}(n \geqslant 2)$. Then there is a sequence of disjoint closed balls $B_{m}$ of radius $r_{m}<\frac{1}{2} r(m=1,2, \ldots)$ lying in the interior of $B$ and covering almost all of $B$.

Proof. This is immediate from the Vitali covering theorem which is stated in $\$ 5$ in connection with the application mentioned in the Introduction.

STEP 2. Let $B$ be a closed ball of radius $r>0$ in $E^{n}(n>2)$. Suppose that $x$ and $y$ are distinguished points on the boundary of $B$ and $\gamma$ is a number satisfying $0<\gamma<1$. Then there is a finite chain of successively tangent, but otherwise disjoint, closed balls $B_{m}$ of radius $r_{m}<\frac{1}{2} r(m=1,2, \ldots, N)$ which lie in the interior of $B$, except that $B_{1}$ touches $x$ and $B_{N}$ touches $y$, and satisfy $\sum_{m=1}^{N} \mu^{n}\left(B_{m}\right)>\gamma \mu^{n}(B)$.

Proof. Take $M_{1}$ balls of total $n$-measure greater than $\gamma \mu^{n}(B)$ from the beginning of the covering of Step 1. Draw a simple curve in the interior of $B$ from $x$ once through each of these $M_{1}$ disjoint balls to $y$. Add $M_{2}$ small balls 
along the curve from $x$ to $y$ like beads on a string to complete the chain of $N=M_{1}+M_{2}$ successively tangent balls.

STEP 3. Start with the given ball $B=B_{1}$ and apply the construction of Step 2 with $x=x_{1}$ and $y=y_{1}$ on the boundary of $B_{1}$ and $\gamma=\gamma_{1}$ satisfying $0<\gamma_{1}<1$. This yields $N=N_{1}$ balls $B_{11}, B_{12}, \ldots, B_{1 N_{1}}$ which may be matched with a partition of $[0,1]=I_{1}$ into $N_{1}$ equal subintervals $I_{11}$, $I_{12}, \ldots, I_{1 N_{1}}$. This ends the first stage of an inductive construction.

At the beginning of the $j$ th stage the balls and corresponding intervals carry $j$-term indices. The range of a particular term in these indices may depend on the preceding terms so we avoid being pedantically explicit. The point is that the successively tangent balls and corresponding intervals are linearly ordered in the natural order of the intervals. The most elegant point of view may be to think of the balls as indexed by the intervals.

To carry out the $j$ th stage, apply the construction of Step 2 to each ball using $x=$ point of contact with predecessor and $y=$ point of contact with successor and using a uniform $\gamma=\gamma_{j}$. Each ball is replaced by a finite chain of new balls and the $j$ th stage is completed by making appropriate equipartitions of the corresponding subintervals of $[0,1]$.

STEP 4. After the $j$ th stage of construction the size of each interval and ball has been reduced by a factor of at least $2^{-j}$ and so the nests which they comprise converge to single points of $[0,1]$ and $B$ respectively. If $q$ is the natural correspondence between these points then $q$ is obviously $1-1$ and continuous. To guarantee $\mu^{n}\left(q^{*}\right)>\alpha \mu^{n}(B)$ it is enough to choose the $\gamma$ 's so that $\prod_{j=1}^{\infty} \gamma_{j}>\alpha$.

The examples of Peano and Osgood were compelling motivation to seek the most general continuous image of $[0,1]$ and the most general homeomorphic image of $[0,1]$. The Hahn-Mazurkiewicz theorem characterizes the Hausdorff topological spaces which are continuous images of $[0,1]$ as the compact, connected, locally connected spaces. A space of this sort is a homeomorphic image of $[0,1]$ if and only if it contains two points which cannot both belong to any proper connected subset. The details of these theorems including references to the original publications may be found in $[1, \S \S 36,39]$. However these results lead away from the objective of the present paper. An early reference which is closer to the spirit of $\$ 2$ is [4].

3. Proof of Theorem 1. Let $d \geqslant 3$ and let $n=d-1$. In order to construct a space curve that points everywhere in $E^{d}$, begin with a Peano curve $p$ : $[0,1] \rightarrow E^{n}$ which covers the unit $n$-cube, $[0,1]^{n}$.

If $B^{n}=\left\{x \in E^{n}:\|x\| \leqslant \sqrt{n /(n+2)}\right\}$ is a ball of radius $\sqrt{n /(n+2)}$ centred at the origin in $E^{n}$, then $[0,1]^{n}$ is homeomorphic to $B^{n}$. Let $h$ : $[0,1]^{n} \rightarrow B^{n}$ be any such homeomorphism and let $u:[0,1] \rightarrow B^{n}$ be the composite mapping $u=h \circ p$. 
The stereographic projection $s: E^{n} \rightarrow S^{n}$ is continuous and carries the ball $B^{n} \subset E^{n}$ onto a cap $C^{n} \subset S^{n}$ which lies in the southern hemisphere of $S^{n}$ and has angular radius arccos $d^{-1}$. This is just the right size of cap to cover one face of an appropriately oriented regular $d$-simplex $T^{d}$ inscribed in $S^{n}$. Recall the mapping $u:[0,1] \rightarrow B^{n}$ and define $v:[0,1] \rightarrow C^{n}$ by $v=s \circ u$.

Regard $v$ as a velocity in $E^{d}$ and obtain part of the space curve $r$ : $[0,1] \rightarrow E^{d}$ as the trajectory of a particle moving with this velocity. That is, define $r(t)=\int_{0}^{t} v(\tau) d \tau$. Then it is obvious that $r:[0,1] \rightarrow E^{d}$ is a continuously differentiable curve and, since $r^{\prime}=v$, it points at least once in every direction belonging to the cap $C^{n}$.

To get a curve that points at least once in every direction, repeat the above construction $d+1$ times, once for each face of the $d$-simplex $T^{d}$, and then link these pieces of curve together cyclically. If these splices are smooth, this leads to a continuously differentiable closed curve in $E^{d}$ that points at least once in every direction. However the objective is to produce a simple curve with these properties, and a few remarks are required to prove that self intersections can be avoided.

Recall that $r$ is the trajectory of a particle travelling for unit time with velocity $v$ and $v$ is a unit vector in the cap $C^{n}$ of angular radius $\arccos d^{-1}$ lying in the southern hemisphere of $S^{n}$. This means that the particle has "downward speed" $v_{D}$ satisfying $d^{-1} \leqslant v_{D} \leqslant 1$ and "horizontal speed" $v_{H}$ satisfying $0 \leqslant v_{H} \leqslant d^{-1}\left(d^{2}-1\right)^{1 / 2}$. The steady downward drift $v_{D} \geqslant d^{-1}>$ 0 guarantees that $r$ does not intersect itself, and the upper bounds $v_{D} \leqslant 1$ and $v_{H} \leqslant d^{-1}\left(d^{2}-1\right)^{1 / 2}$ guarantee that $r$ is confined to a cylinder of length 1 and radius $d^{-1}\left(d^{2}-1\right)^{1 / 2}$.

The $d+1$ pieces of curve belonging to the different faces of the simplex $T^{d}$ are each simple curves. If their confining cylinders are translated so that they become mutually disjoint, then the different pieces of curve do not intersect one another. With this arrangement of the important pieces, it is easy to add the cyclic splices and produce a simple, closed curve as required.

Finally, Theorem 1 asserts that if $r:[a, b] \rightarrow E^{d}(d \geqslant 3)$ is continuously differentiable and $r^{\prime}$ points at least once in every direction $S^{d-1}$ then $r^{\prime}$ must point at least $d$ times in certain directions. The set of points where $r^{\prime} \neq 0$ is open, hence a countable union of open intervals. Each of these is a countable union of closed intervals and so there exists a countable collection of continuous functions $\rho_{j}=r^{\prime} /\left\|r^{\prime}\right\|: J_{j} \rightarrow S^{d-1}$ defined on closed intervals and together covering $S^{d-1}$. According to Lebesgue [5] there is a function $\rho_{j}$ and a point $z \in S^{d-1}$ such that $\rho_{j}^{-1}(z)$ contains $(d-1)+1=d$ points. It follows that $r^{\prime}$ points in the same direction at these $d$ different times.

4. Proof of Theorem 2. Theorem 2 asserts inter alia that if $r:[a, b] \rightarrow E^{d}$ $(d>3)$ is continuously differentiable and $r^{\prime} \neq 0$ and $\rho=r^{\prime} /\left\|r^{\prime}\right\|:[a, b] \rightarrow$ 
$S^{d-1}=S^{n}$ is injective then $\rho^{*}=\rho([a, b])$ cannot be almost all of $S^{n}$. The set $\rho^{*}$ is homeomorphic to $[a, b]$ and therefore it must be a proper subset of $S^{n}$. Since $\rho^{*}$ is also compact, $S^{n}-\rho^{*}$ is a nonvoid open set and therefore a set of positive $n$-dimensional measure.

Still in the situation $r^{\prime} \neq 0$, Theorem 2 asserts that there is a simple, closed, continuously differentiable curve $r:[a, b] \rightarrow E^{d}(d \geqslant 3)$ such that $\rho=$ $r^{\prime} /\left\|r^{\prime}\right\|:[a, b] \rightarrow S^{d-1}=S^{n}$ is injective and $\mu^{n}\left(\rho^{*}\right)$ is arbitrarily close to $\mu^{n}\left(S^{n}\right)$. The proof of this involves Lemma 1 and a modified version of the methods of Theorem 1 .

Let $B^{n}=\left\{x \in E^{n}:\|x\| \leqslant 1-\varepsilon\right\}$ where $0<\varepsilon<1$; let $\alpha$ satisfy $0<\alpha<$ 1 ; let $x$ and $y$ be diametrically opposite points of $B^{n}$ and let $v_{0}$ and $v_{1}$ be points outside of $B^{n}$ on the line $x y$ such that $\left\|v_{0}-x\right\|=\left\|v_{1}-y\right\|=\varepsilon$. Let $0<\delta<1$ and apply Lemma 1 to obtain $q:[(1-\delta) / 2,(1+\delta) / 2] \rightarrow B^{n}$ such that $q((1-\delta) / 2)=x, q((1+\delta) / 2)=y$ and the rest of $q^{*}$ lies in the interior of $B^{n}$ and satisfies $\mu^{n}\left(q^{*}\right) \geqslant \alpha \mu^{n}\left(B^{n}\right)$. Extend $q$ : [(1- $\left.\delta\right) / 2,(1+$ $\delta) / 2] \rightarrow B^{n}$ to $u:[0,1] \rightarrow E^{n}$ so that $u$ maps $[0,(1-\delta) / 2]$ linearly onto the segment between $u(0)=v_{0}$ and $u((1-\delta) / 2)=x$ and $u$ maps $[(1+\delta) / 2,1]$ linearly onto the segment between $u((1+\delta) / 2)=y$ and $u(1)=v_{1}$. The stereographic projection $s: E^{n} \rightarrow S^{n}$ fixes $v_{0}$ and $v_{1}$ and pushes the rest of $u^{*}$ into the southern hemisphere of $S^{n}$ and $q^{*} \subset u^{*}$ into a cap centred at the south pole with angular radius $2 \arctan (1-\varepsilon)$. As in Theorem 1 , define $v=s \circ u$ and $r:[0,1] \rightarrow E^{d}$ by $r(t)=\int_{0}^{t} v(\tau) d \tau$.

It follows that $r$ is a continuously differentiable curve and $r^{\prime}=v$ never points twice in the same direction. Since $v^{*}$ includes $s\left(q^{*}\right), \mu^{n}\left(v^{*}\right) \rightarrow \frac{1}{2} \mu^{n}\left(S^{n}\right)$ as $\varepsilon \rightarrow 0^{+}$and $\alpha \rightarrow 1^{-}$. The velocities $v(0)=v_{0}$ and $v(1)=v_{1}=-v_{0}$ are horizontal but otherwise $v$ points downward, so that $r$ is a simple curve. The velocities $v(t)$ and $v(1-t), 0 \leqslant t \leqslant(1-\delta) / 2$, have the same downward component, so a horizontal $(d-1)$-flat through the point $\frac{1}{2}(r(0)+r(1))$ separates the corresponding legs of the trajectory and cuts $r^{*}$ at $r\left(t_{0}\right)$ for some $(1-\delta) / 2<t_{0}<(1+\delta) / 2$.

Extend the domain of $v$ from $[0,1]$ to $[0,2]$ by setting $v(1+t)=-v(t)$ for $0 \leqslant t \leqslant 1$. This gives a continuous function $v:[0,2] \rightarrow S^{n}$ such that $v(t)$ lies in the northern hemisphere if $1 \leqslant t \leqslant 2$. Use the extended definition of $v$ together with the old formula $r(t)=\int_{0}^{t} v(t) d \tau$ to obtain an extended function $r:[0,2] \rightarrow E^{d}$. It follows that $r$ is a continuously differentiable curve, $r^{\prime}=v$ never points twice in the same direction and $\mu^{n}\left(v^{*}\right) \rightarrow \mu^{n}\left(S^{n}\right)$ as $\varepsilon \rightarrow 0^{+}$and $\alpha \rightarrow 1^{-}$. The fact that $v(1+t)=-v(t)$ if $0 \leqslant t \leqslant 1$ yields

$$
r(1+t)-r(1)=-[r(t)-r(0)]
$$

or

$$
r(1+t)=[r(0)+r(1)]-r(t)
$$


Geometrically, this means that $r(1+t)$ is the image of $r(t)$ under reflection in the point $\frac{1}{2}[r(0)+r(1)]$. This guarantees that $r$ is a closed curve and $r$ is simple on $[1,2]$ as well as on $[0,1]$.

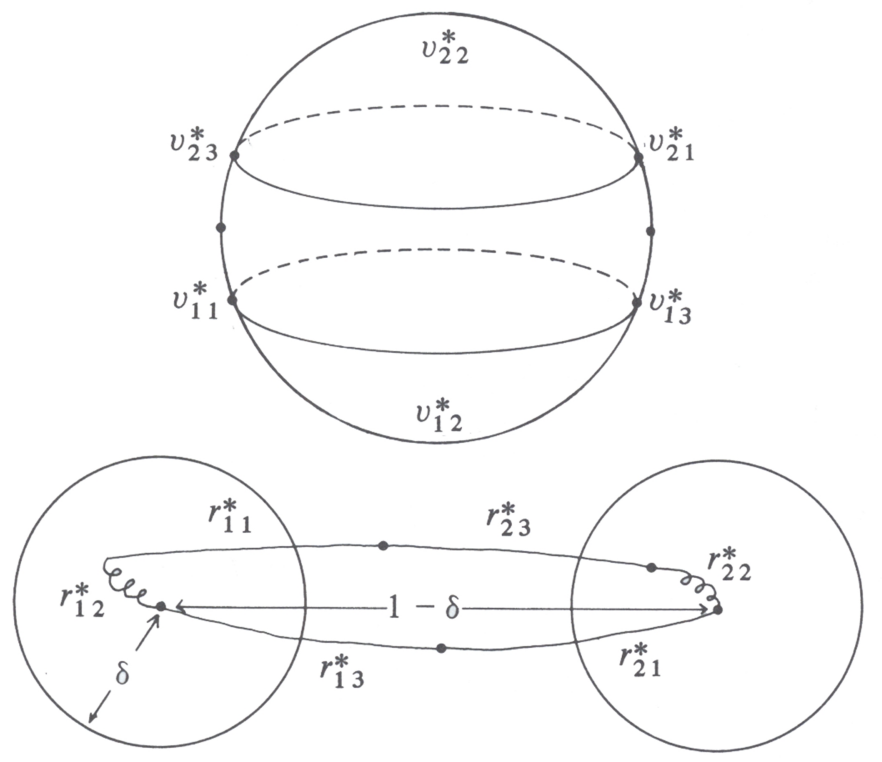

FIGURE 2

To complete the proof of this part of Theorem 2 it remains to show that $r\left(t_{1}\right)=r\left(t_{2}\right)$ cannot hold with $0<t_{1}<1<t_{2}<2$. Let $I_{11}=[0,(1-\delta) / 2]$, $I_{12}=[(1-\delta) / 2,(1+\delta) / 2], \quad I_{13}=[(1+\delta) / 2,1]$ and $I_{2 j}=I_{1 j}+1 \quad(j=$ $1,2,3)$. If $t_{1} \in I_{11}$ or $I_{13}$ and $t_{2} \in I_{21}$ or $I_{23}$ respectively then $r\left(t_{1}\right)$ and $r\left(t_{2}\right)$ lie on opposite sides of the horizontal $(d-1)$-flat through $\frac{1}{2}[r(0)+r(1)]$. If $t_{1} \in I_{13}$ and $t_{2} \in I_{21}$, then intersections are also impossible because, for $(1+\delta) / 2 \leqslant t \leqslant(3-\delta) / 2,\left\|r^{\prime}(t)-v_{1}\right\| \sim \varepsilon$, so that $r(t)$ practically traces a line segment, $l_{1}$, of length $1-\delta$ parallel to $v_{1}$. Similarly there is no problem if $t_{1} \in I_{11}$ and $t_{2} \in I_{23}$ because, for $t \in I_{23} \cup I_{11}, r(t)$ practically traces a line segment, $l_{0}$, of length $1-\delta$ parallel to $v_{0}=-v_{1}$. Finally, the complicated motions for $t_{1} \in I_{12}$ and $t_{2} \in I_{22}$ take place in balls of radius $\delta$ and bridge the gap from the leading end of $l_{0}$ to the trailing end of $l_{1}$ and vice versa. If $\delta<1 / 5$ there is no possibility of unwanted intersections as $\varepsilon \rightarrow 0^{+}$. Figure 2 is a schematic illustration of the situation.

5. Completion of the proof of Theorem 2. The last part of Theorem 2 asserts that there exists a simple, closed, continuously differentiable curve in $E^{d}$ $(d \geqslant 3)$ whose tangent vector points no more than once in any direction on 
$S^{d-1}$ yet only omits a set of directions of $(d-1)$-dimensional measure 0 .

The proof of this uses the Vitali covering theorem [2]. The basic notation and definitions are as follows.

Let $(X, d)$ be a compact metric space. A subset $F \subset X$ has diameter

$$
\delta(F)=\sup \{d(x, y): x \in F \text { and } y \in F\}
$$

and $\varepsilon$-neighbourhood

$$
N(F, \varepsilon)=\{x \in X: d(F, x) \leqslant \varepsilon\} .
$$

Let $\mu$ be a complete measure defined on a $\sigma$-algebra containing the Borel subsets of $X$. A family of closed sets $\mathscr{F}=\{F\}$ is said to cover $X$ in the sense of Vitali if

(i) each $F \in \mathscr{F}$ has positive $\mu$-measure, and

(ii) there is a constant $1<k<\infty$ such that each point $x \in X$ is contained in elements $F \in \mathscr{F}$ of arbitrarily small diameter satisfying $\mu(N(F, 3 \delta(F))) / \mu(F) \leqslant k$.

Vitali Covering Theorem. If $\mathcal{F}=\{F\}$ is a Vitali covering of $X$ then there is a sequence $\left\{F_{j}\right\}$ of disjoint elements of $\mathscr{F}$ such that $\mu\left(X-U F_{j}\right)=0$.

In $\S 2$, Lemma 1 , Step 1 this theorem is used with $X=B=\left\{x \in E^{n}\right.$ : $\|x\| \leqslant r\}, \mu=\mu^{n}$ and $\mathcal{F}=$ family of all closed balls contained in $B$ with radius $\leqslant \frac{1}{2} r$. Strictly speaking it appears that some of the balls selected by the theorem might touch the boundary of $B$ instead of lying in its interior. However the details of the Vitali proof show that this can be avoided.

In this section the Vitali covering theorem is used with $X=S^{n}=\{z \in$ $\left.E^{n+1}:\|z\|=1\right\}, \mu=\mu^{n}$ and $\mathscr{F}=\left\{f^{*}\right\}$, where the functions $f:[0,1] \rightarrow S^{n}$ are constructed from Osgood curves much as in $\$ 4$. A typical function of this type is $f=g \circ s \circ q$, where $q:[0,1] \rightarrow B^{n}, B^{n}$ of radius $r$, is an Osgood curve with $\mu^{n}\left(q^{*}\right) \geqslant \alpha \mu^{n}\left(B^{n}\right) ; s: B^{n} \rightarrow S^{n}$ is stereographic projection and $g: S^{n} \rightarrow$ $S^{n}$ is a rotation. If $g \circ s\left(B^{n}\right)=C^{n}(\theta)$, a cap of angular radius $\theta$, then $f^{*} \subset C^{n}(\theta)$ and $N\left(f^{*}, 3 \delta\left(f^{*}\right)\right) \subset C^{n}(3 \theta)$. If $\alpha$ is close to $1, \mu^{n}\left(f^{*}\right)$ is close to $\mu^{n}\left(C^{n}(\theta)\right)$ and it is safe to assume $\mu^{n}\left(f^{*}\right)>\frac{1}{2} \mu^{n}\left(C^{n}(\theta)\right)$. Under these circumstances the Vitali ratio satisfies

$$
\mu^{n}\left(N\left(f^{*}, 3 \delta\left(f^{*}\right)\right)\right) / \mu^{n}\left(f^{*}\right) \leqslant 2 \mu^{n}\left(C^{n}(3 \theta)\right) / \mu^{n}\left(C^{n}(\theta)\right)<2 \cdot 3^{n}=k .
$$

Moreover, if $r$ is small, then $\theta$ is small and $\delta\left(f^{*}\right)$ is small, so that $\mathscr{F}=\left\{f^{*}\right\}$ really does give a Vitali covering of $S^{n}$.

A Vitali covering is not spoiled by discarding its larger sets. Moreover, finitely many members of the disjoint sequence guaranteed by the theorem may be selected arbitrarily. Lemma 2 is proved by applying the Vitali covering theorem, strengthened by these remarks, to the cover $\mathscr{F}=\left\{f^{*}\right\}$ of $S^{n}$. 
LEMMA 2. There is a countable collection of continuous injections $f_{j}:[0,1] \rightarrow$ $S^{n}(n>2)$ with the following properties:

(i) The image sets $f_{j}^{*}(j=1,2, \cdots)$ are disjoint and $\mu^{n}\left(S^{n}-U f_{j}^{*}\right)=0$.

(ii) $f_{j}^{*} \subset C_{j}$ where $C_{j}$ is a cap with centre $c_{j}$ and angular radius $\theta_{j}<\Pi / 2$ $\arccos (n+1)^{-1}$.

(iii) For $k=1,2, \ldots, n+2$ the caps $C_{2 k}$ and $C_{2 k-1}$ of (ii) are contained in caps $E_{k}$ with centre $e_{k}$ and angular radius $\psi$ where the $e_{k}$ are the centroids of the faces of a regular simplex inscribed in $S^{n}$.

The functions $f_{j}:[0,1] \rightarrow S^{n}$ of Lemma 2 are modified to functions

$$
b_{j}:[0,1] \rightarrow B^{n+1}=\left\{z \in E^{n+1}:\|z\|<1\right\}
$$

by setting $b_{j}(t)=t(1-t) f_{j}(t)$. The functions $b_{j}$ are bursts of velocity giving rise to displacement $d_{j}=\int_{0}^{1} b_{j}(\tau) d \tau$ satisfying

(i) $\left\|d_{j}\right\|<\int_{0}^{1} \tau(1-\tau) d \tau=1 / 6$,

(ii) $d_{j} \cdot c_{j} \geqslant \int_{0}^{1} \tau(1-\tau) \cos \theta_{j} d \tau=(1 / 6) \cos \theta_{j}$, and

(iii) $d_{j} \cdot e_{k} \geqslant(1 / 6) \cos \psi(j=2 k-1,2 k ; k=1,2, \ldots, n+2)$.

When time is rescaled to an interval of length $\delta_{j}>0$ these bursts of velocity take the form

$$
v_{j}:\left[\alpha_{j}, \beta_{j}\right] \rightarrow E^{n+1}, \quad v_{j}(t)=b_{j}\left(\delta_{j}^{-1}\left[t-\alpha_{j}\right]\right), \quad \text { where } \beta_{j}-\alpha_{j}=\delta_{j},
$$

and give rise to simple trajectories

$$
r_{j}:\left[\alpha_{j}, \beta_{j}\right] \rightarrow E^{n+1}, \quad r_{j}(t)=r_{j}\left(\alpha_{j}\right)+\int_{\alpha_{j}}^{t} v_{j}(\tau) d \tau
$$

of total displacement

$$
\begin{aligned}
r_{j}\left(\beta_{j}\right)-r_{j}\left(\alpha_{j}\right) & =\int_{\alpha_{j}}^{\beta_{j}} v_{j}(\tau) d \tau=\int_{\alpha_{j}}^{\beta_{j}} b_{j}\left(\delta_{j}^{-1}\left[\tau-\alpha_{j}\right]\right) d \tau \\
& =\delta_{j} \int_{0}^{1} b(u) d u=\delta_{j} d_{j} .
\end{aligned}
$$

If $\sum_{j=1}^{\infty} \delta_{j}=1$ the time intervals $\left[\alpha_{j}, \beta_{j}\right]$ may be packed without interior overlap to fill $[0,1]$. Since the functions $v_{j}$ vanish at the endpoints of their domains, the function $v:[0,1] \rightarrow E^{n+1}$ defined by its restrictions, $v \|\left[\alpha_{j}, \beta_{j}\right] \equiv$ $v_{j}$, is continuous. It follows that $r:[0,1] \rightarrow E^{n+1}$ defined by $r(t)=\int_{0}^{t} v(\tau) d \tau$ is continuously differentiable with $r^{\prime}=v$. The tangent vector $v$ points at most once in any direction of $\boldsymbol{S}^{n}$ yet only omits a set of directions with $n$-dimensional measure 0 . This follows from Lemma 2(i) because, except for the countably many points where $v$ vanishes,

$$
\left(\frac{v}{\|v\|}\right)^{*}=\bigcup_{j}\left(\frac{v_{j}}{\left\|v_{j}\right\|}\right)^{*}=\bigcup_{j}\left(\frac{b_{j}}{\left\|b_{j}\right\|}\right)^{*}=\bigcup_{j} f_{j}^{*} .
$$


It remains to choose the $\delta_{j}>0$ and to order the intervals $\left[\alpha_{j}, \beta_{j}\right]=I_{j}$ so that $r$ becomes a simple, closed curve.

The vectors $e_{k}$ of Lemma 2(iii) form a positive basis for $E^{n+1}$ and satisfy $\sum_{k=1}^{n+2} e_{k}=0$. When $\psi$ is sufficiently small the two sets of $n+2$ vectors $\left\{d_{2 k-1}\right\}$ and $\left\{d_{2 k}\right\}$ each form a positive basis for $E^{n+1}$ and each represent 0 with coefficients arbitrarily close to 1 . In order to pick appropriate $\delta_{j}$ 's, let $\delta_{j}^{\prime}=2^{2 n+4-j}(j=2 n+5,2 n+6, \cdots) ;$ construct $d=\sum_{j=2 n+5}^{\infty} \delta_{j}^{\prime} d_{j}$ and represent $-d$ in the form $-d=\sum_{j=1}^{2 n+4} \delta_{j}^{\prime} d_{j}$ with $\delta_{j}^{\prime} \sim \lambda>0 \quad(j=$ $1,2, \ldots, 2 n+4)$. Then if $\delta_{j}=\delta_{j}^{\prime}\left[\sum_{i=1}^{\infty} \delta_{i}^{\prime}\right]^{-1}$, it follows that

$$
\begin{aligned}
& \text { (i) } \sum_{j=1}^{\infty} \delta_{j}=1, \\
& \text { (ii) } \sum_{j=1}^{\infty} \delta_{j} d_{j}=0 \text { and } \\
& \text { (iii) } \delta_{l} \sim \lambda \sum_{j=2 n+5}^{\infty} \delta_{j} \text { provided } 1<l<2 n+4 .
\end{aligned}
$$

The first condition fulfills an obligation incurred in the preceding paragraph; the second condition insures that $r$ is a closed curve because $r(1)-r(0)=$ $\sum_{j=1}^{\infty} \delta_{j} d_{j}$ and the third condition is useful in arranging for $r$ to be a simple curve. This last task will be accomplished by making $r$ very close to the simple polygon $P$ with vertices $0, \mu e_{1}, \mu\left(e_{1}+e_{2}\right), \ldots, \mu\left(e_{1}+e_{2}+\cdots+e_{n+2}\right)=$ 0 , where $\mu=1 / 6(n+2)$.

Let $F_{k}$ be the cap with centre $e_{k}$ and angular radius arccos $(n+1)^{-1}$, the circumradius of a face of the regular $(n+1)$-simplex inscribed in $S^{n}$. Since the $F_{k}$ cover $S^{n}$, it is possible to partition the positive integers $j=1,2, \ldots$ into sets $J_{k}(k=1,2, \ldots, n+2)$ in such a way that if $j \in J_{k}$ then $c_{j} \in F_{k}$. There is some choice in this partition, but when $\psi$ is small, $2 k-1$ and $2 k$ must belong to $J_{k}$. Order the elements of each $J_{k}$ by starting with $2 k-1$, finishing with $2 k$ and taking the other elements in their natural order. Then order the positive integers by taking the $J_{k}$ in their natural order. If the time intervals $I_{j}$ are packed into $[0,1]$ in this order, $r$ is a simple curve.

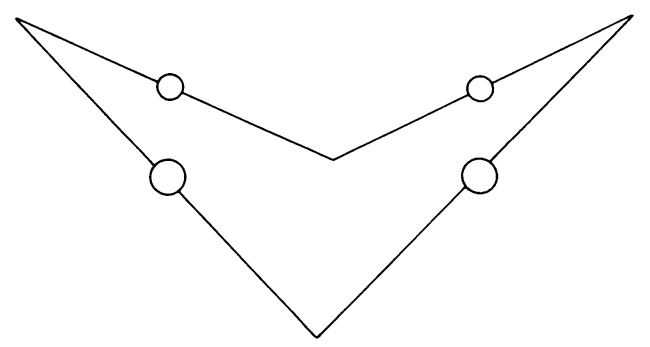

FIGURE 3 
On the $k$ th "edge" of $r$, where $j \in J_{k}, r_{j}^{\prime} \cdot e_{k}>0$ because $\theta_{j}<\Pi / 2$-arccos $(n+1)^{-1}$ as specified in Lemma 2(ii). This means that there is steady drift in the $e_{k}$ direction and no chance for unwanted intersections. The first and last steps of this edge are $\delta_{l} d_{l}$ with $l=2 k-1$ and $2 k$ respectively, and these become arbitrarily close to $\frac{1}{2} \mu e_{k}$ as $\psi \rightarrow 0$ making $d_{l} \rightarrow e_{k} / 6$ by (A)(i), (iii) and $\lambda \rightarrow \infty$ making $\delta_{l} \rightarrow 1 /(2 n+4)$ by (B)(i), (iii). The middle steps $\Sigma\left\{\delta_{j} d_{j}\right.$ : $\left.j \in J_{k}-\{2 k-1,2 k\}\right\}$ have total length at most

$$
\frac{1}{6} \sum_{j=2 n+5}^{\infty} \delta_{j} \leqslant \frac{1}{6 \lambda} \delta_{2 k}<\frac{\mu}{\lambda}
$$

and so they fit into an arbitrarily small ball as $\lambda \rightarrow \infty$. This has the double benefit of making the total displacement on the $k$ th edge arbitrarily close to

$$
\delta_{2 k-1} d_{2 k-1}+\delta_{2 k} d_{2 k} \rightarrow \mu e_{k}
$$

and of keeping these middle steps out of the way of all other steps when the edges of $r$ are put together in close approximation to $P$. Figure 3 illustrates the situation in dimension 3 .

6. Related work. Two other papers related to this one discuss the possibility of packing $(d-1)$-balls in $E^{d}(d \geqslant 2)$. The first paper [8] shows that it is possible to choose $c$ different directions and, perpendicular to each of these directions, $c$ congruent $(d-1)$-balls in such a way that the entire collection remains pairwise disjoint. The second paper [9] shows that it is not possible to go too far in this direction without forcing overlap, even if the $(d-1)$-balls are allowed to be of different sizes. The precise statement of the constraint is developed in the next paragraph.

Suppose that a collection of $(d-1)$-balls is given in $E^{d}(d>2)$. For each direction $v \in S^{d-1}$ let $l(v)$ be the line through the origin with direction $v$. Let $S(v) \subset l(v)$ be the set of points where $l(v)$ meets $(d-1)$-flats which are perpendicular to $v$ and carry a $(d-1)$-ball of the given collection. For each real number $p$ with $0 \leqslant p \leqslant 1$ let $V(p)=\left\{v \in S^{d-1}\right.$ : Hausdorff $\operatorname{dim} S(v)$ $\geqslant p$ \} and let $q(p)=$ Hausdorff $\operatorname{dim} V(p)$. Then, if the given $(d-1)$-balls are pairwise disjoint, it follows that for each $p, p+q(p)<1$. Two different extreme situations, $q(1)=0$ and $q(0)=1$, can be visualized by fattening a line into a cylinder and a circle into a torus and considering the $(d-1)$-balls which arise as perpendicular sections of the fattened objects.

The second example points to a distinction between the circle in $E^{2}$ and the various analogues which we have constructed in $E^{d}(d>3)$. In each case one can imagine the curve fattened to a tubular neighbourhood and this neighbourhood foliated by $(d-1)$-dimensional sections perpendicular to the curve's tangent. When $d=2$ this works perfectly to give an annulus foliated by line segments. But when $d>2$ the leaves of the alleged foliation satisfy 
$q(0)=d-1>1$ and there are wild intersections even when the tubular neighbourhood is not of uniform width.

The connection between the present paper and the first one mentioned above is somewhat more technical. Suffice it to say that the construction of $c$ directions with $c(d-1)$-balls each depends on the construction of a Lipschitz function which assumes $c$ values each of which is taken on $c$ times. This construction can be effected by modifying the construction of Peano curves given in $\$ 2$. Instead of interpreting $x(t)=\cdot t_{1} t_{3} \ldots$ in a lower scale to make it take on all values, we interpret it in a higher scale to smooth it.

\section{REFERENCES}

1. F. Hausdorff, Set theory, Chelsea, New York, 1957.

2. L. L. Helms, Introduction to potential theory, Wiley, New York, 1969.

3. D. Hilbert, Ueber die stetige Abbildung einer Linie auf ein Flächenstück, Math. Ann. 38 (1891), 459-460.

4. K. Knopp, Einheitliche Erzeigung und Darstellung der Kurven von Peano, Osgood und v. Koch, Arch. Math. 26 (1918), 103-115.

5. H. Lebesgue, Sur les correspondences entre les points de deux espaces, Fund. Math. 2 (1921), 256-285.

6. W. F. Osgood, A Jordan curve of positive area, Trans. Amer. Math. Soc. 4 (1903), 107-112.

7. G. Peano, Sur une courbe, qui remplit toute une aire plane, Math. Ann. 36 (1890), 157-160.

8. J. B. Wilker, Russian dolls, Canad. Math. Bull. 21 (1978), 237-240.

9. , Hausdorff obstructions to packing ( $N-1$ )-balls in $N$-space (to appear).

Department of Mathematics, The australian national University, P. O. Box 4, Canberra, A. C. T. 2600, Australia

Current address: Department of Mathematics, University of Toronto, Toronto, Ontario, Canada 